Editorial

\title{
Effort testing in child cognitive and neuropsychological testing
}

Volume 5 Issue 5 - 2016

Robert Perna

Clinical neuropsychologist, TIRR Memorial Hermann, USA

\section{Editorial}

Assessing clinicians often understand the need to utilize reliable tests that are validated for a specific purpose and population and which have various norms that should be selected for appropriateness; however, many pediatric clinicians don't understand the need for effort testing. Effort testing has become, in some peoples view, equal to testing for malingering. These measures are much more than that. Empirical research clearly shows that children do not always provide adequate effort on cognitive and neuropsychological testing. Effort testing in the context of cognitive or neuropsychological testing serves the purpose of allowing the clinician to be reasonably confident that sufficient effort was provided by the examinee and therefore the acquired data is likely accurately representative of the persons cognitive functioning at that time. Adults and children have been shown to provide inconsistent effort on various tests and assessments. Considerable research suggests that behavioral observations do not reliably allow a clinician to know if a full effort was provided, except for extremely obvious cases. Though most psychologists and neuropsychologists administer effort tests as part of their evaluation procedures, many who work with children continue to feel this is unneeded. A consequence of this situation is that inadequate effort on testing can result in an abnormal score profile and perhaps incorrect conclusions regarding impairments and diagnoses. Some empirical research $^{1}$ has found the when neuropsychologists assess children that were instructed to malinger, the neuropsychologists were inclined to believe that adequate effort was provided. Specifically, $78 \%$ to $83 \%$ of neuropsychologists in these studies interpreted malingering children's test scores as impaired and reported a high level of confidence in their conclusions.

\begin{abstract}
Correspondence: Robert Perna, Clinical neuropsychologist, TIRR Memorial Hermann, Texas Medical Center Houston, 2450 Holcombe Blvd \#I, Houston, TX 7702I, USA, Tel 706-750-2572, Email drperna@juno.com
\end{abstract}

Received: October 27, 2016 | Published: November 03, 2016

A cost benefit analysis will always favor the administration of at least one appropriate performance validity test as part of an assessment battery.

\section{Acknowledgments}

None.

\section{Conflicts of interest}

Author declares that there is no conflict of interest.

\section{References}

1. Walker JS. Malingering in Children: Fibs and faking. Child Adolesc Psychiatr Clin N Am. 2011;20(3):547-556. 